\title{
Development of visual attention: A stereoscopic view
}

\author{
CLAUDIA L. HEINBUCK and WAYNE A. HERSHBERGER \\ Northern Illinois University, De Kalb, Illinois
}

\begin{abstract}
Five-year-olds, 8-year-olds, and adults (20 each) tachistoscopically viewed random-dot stereograms containing either uniform depth or an elevated disk whose perimeter fell $1.5^{\circ}, 2.5^{\circ}$, or $4^{\circ}$ beyond fixation. When subjects were forewarned as to the size of the disk to look for (precued trials), signal detection improved ( $d^{\prime}$ increased) for all three groups, and 5-year-olds improved the most. Without forewarning (self-cued trials), the 5-year-olds performed more poorly than the other two groups, which performed alike. Although young children are able to allocate their visual attention selectively, they do not appear to deploy it as quickly or as effectively as their elders when the focus of attention is theirs to choose.
\end{abstract}

We assessed age-related differences in visual selective attention (aiming visual attention in one direction while gazing in another) under conditions of tachistoscopic exposure. The evidence for age-related differences in visual attention during tachistoscopic exposure comes from experiments that required subjects to read (Taylor, 1982) or compare letters of the alphabet (Fisher \& Lefton, 1976). Conceivably, the observed age-related differences in those studies reflected the development of reading rather than attention. In the present study, we utilized an innate and developmentally mature mechanism, stereopsis (Julesz, 1971), rather than an acquired skill such as reading.

Helmholtz (1925/1962)was the first to note the effect of visual selective attention upon stereopsis. He juxtaposed a pair of stereoscopic pictures on an inside wall of a dark box and placed a pinhole, which was illuminated from the rear, in the center of each picture. Looking into the box through a hole in the opposite wall, Helmholtz focused his left and right eyes upon the left and right pinholes, respectively, until the pinholes appeared to fuse as one. A brief spark of light was then discharged, and he obtained an impression of stereoscopic relief (stereopsis), depending upon his visual attention. In Helmholtz's words, "the observer may be gazing steadily at the two pinholes and holding them in exact coincidence, and yet at the same time he can concentrate his attention on any part of the dark field he likes, so that when the spark comes, he will get an impression about objects in that particular region only" (Helmholtz, 1925/1962, p. 455).

Subjects in the present experiment viewed random-dot stereograms (Julesz, 1971) in a tachistoscopic stereoscope similar to Helmholtz's. Each stereogram depicted a flat gray square surface (the random-dot array) that either was empty or incorporated a single elevated disk centered upon

This report is based on the doctoral dissertation of the first author. Requests for reprints should be sent to Claudia Heinbuck, Department of Arts and Sciences, Macomb Community College, 44575 Garfield Rd., Mt. Clemens, MI 48044. the square. The size of the random-dot array was varied from trial to trial, and the size of the disk varied accordingly. The subject's task in each trial was to detect the presence or absence of the disk. Since the size of the disk varied with the size of the random-dot array, the size of the array provided a salient cue as to the approximate size of the disk to be detected, a cue that became available to the subject as soon as the stereogram was exposed. On some trials (self-cued), no other cues were provided. On other trials (precued), the subject was also shown the size of disk to look for during that trial.

The precued trials were designed to favor subjects differentially according to their ability to aim either their gaze or their attention accurately at the informative regions of the stereogram (i.e., points along the disk's perimeter) while keeping the other (gaze or attention) fixed upon a pair of dichoptic fixation points. (The subjects began each trial by perceptually fusing a pair of fixation dots equivalent to Helmholtz's two pinholes.) The self-cued trials allowed subjects to aim their gaze accurately only at the fused fixation points, and differentially favored subjects according to their ability to shift their attention quickly to the disk's perimeter once the trial had begun.

Although both types of trials were designed to favor subjects differentially according to their ability selectively to deploy their visual attention (i.e., look one direction while gazing another), the self-cued trials called for a much greater facility in this regard than did the precued trials. In the present experiment, therefore, developmental differences in visual selective attention could have manifested themselves either as a main effect of age or as an age $\times$ cue type interaction, or as both.

We note here a very important feature of Helmholtz's stereoscopic method: it obliges the subject to fuse the dichoptic fixation points in order to be able to detect the stereoscopic figure. The subject cannot enhance his or her signal detection performance by ignoring instructions to fixate the fixation point, as has often been the case in previous developmental investigations of visual attention. 
Rather, the subject is required to perform two visual tasks at once (fixation and signal detection), but at two different retinal locations, one in the periphery and one at the fovea.

Another frequent shortcoming of previous investigations of visual attention has been the confounding of sensitivity and criterion effects (see Muller \& Findlay, 1987). In the present experiment, signal detection theory (Swets, 1973) was used to isolate these two effects, with parameter $d^{\prime}$ reflecting the subject's sensitivity to the signal, and parameter $\beta$ reflecting the stringency of the subject's decision criterion.

\section{METHOD}

\section{Subjects}

Students enrolled in various psychology courses at Macomb Community College (Warren, Michigan) were asked to volunteer themselves and/or to aid in the location of 5- and 8-year-olds. The only stipulation was that the volunteer's visual acuity be normal or corrected to normal with contact lenses. The subject pool consisted of 60 individuals: 20 5-year-olds, 9 males and 11 females, ranging in age from 5 years to 5 years $/ 10$ months, with a mean age of 5 years/5 months; 20 8-year-olds, 14 males and 6 females, ranging in age from 8 years to 8 years $/ 9$ months, with a mean age of 8 years/ 4 months; and 20 college students, 7 males and 13 females, with a mean age of 24 years $/ 6$ months. Two 5 -year-olds had to be dropped from the subject pool because of failure to understand instructions. One 8-year-old was dropped because of suspected amblyopia not apparent during pretesting. Two college students could not participate because of failure to obtain proper binocular fusion.

The subjects participated one at a time. Thirteen of the youngsters were students at Montessori Academy (Warren, Michigan) and were tested at the academy. Five youngsters were tested in a classroom at Macomb Community College, and the remaining children were tested in their own homes. All of the college students were tested in a classroom at the college. None of the subjects had had previous tachistoscopic experience.

\section{Stimuli}

The stimuli (see Figure 1) were six different random-dot stereograms, each comprising a pair of small, medium, or large randomdot arrays $(22,36$, or $57 \mathrm{~mm}$ square, respectively) separated $66 \mathrm{~mm}$ on center. One stereogram of each size had a pair of identical arrays and yielded no stereopsis. The other stereogram of each size incorporated binocular disparity depicting a single elevated disk centered above the rest of the random-dot array. The diameters of the disks in the small, medium, and large arrays were 10,17 , and $27 \mathrm{~mm}$, respectively. As viewed by the subjects, these diameters corresponded to visual angles of $3^{\circ}, 5^{\circ}$, and $8^{\circ}$, respectively. All six stereograms were derived photographically, in either their original or reduced size, from a prototype published by Julesz (1971, p. 272). Altogether, 12 finished photographs were made-2 identical sets of the 6 stereograms described above.

\section{Apparatus}

The photographs were mounted face down in a Brewster stereoscope and transilluminated by a strobe light wired to an electronic timer designed to function as a tachistoscope. Looking into the stereoscope, the subjects saw the back of a photograph whose image became visible only when transilluminated by the strobe light. The retinal image of the stereogram was a bit blurred, but this blur did not prevent stereopsis.

\section{Fixation Dots and Attention Cues}

Two fixation dots were printed on the back of each photograph, $66 \mathrm{~mm}$ apart, corresponding to the centers of the two arrays that
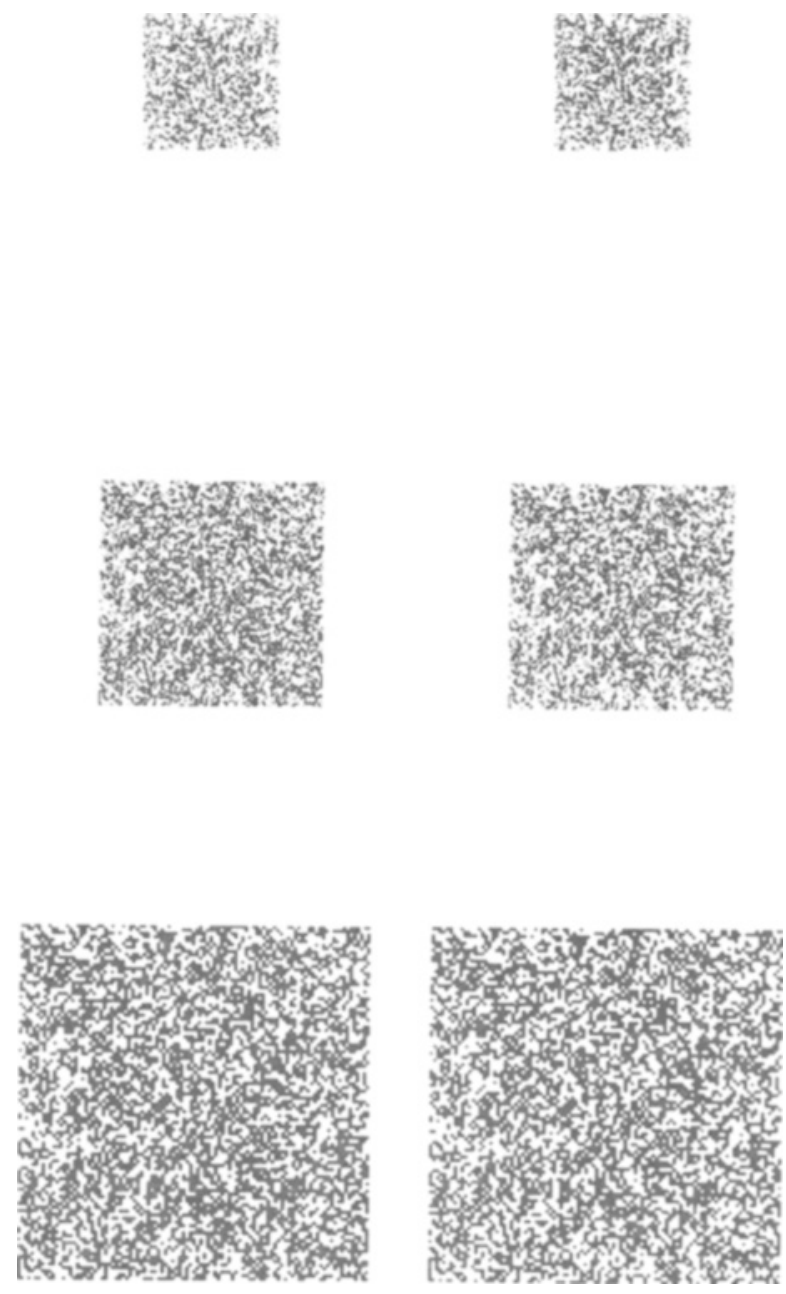

Figure 1. Three random-dot stereograms, each depicting a single elevated disk whose diameter is proportional to the size of the random-dot arrays.

appeared on the front. For one of the two sets of six stereograms described above, the back of each photograph also bore two additional pairs of dots which indicated the size of the disk whose presence or absence was to be detected. One pair of dots was vertically arrayed about the left fixation dot (:); the other pair was horizontally arrayed about the right fixation dot $(\cdots)$. Binocular fusion of the two fixation dots, the only dichoptic stimuli among the six dots, yielded a binocular pattern $(\cdot: \cdot)$, with the four outlying dots locating the perimeter of the disk whose presence or absence was to be detected.

\section{Procedure}

The subjects were tested individually. To introduce them to the task, they were seated in front of the stereoscope and asked to view each of the various photographs displayed face up in the stereoscope, and to report what they saw. Although a wide variety of labels were used (e.g., circle, ball, knob) by different subjects, the detection of the disk figure in all three of its sizes and the report of its absence in appropriate stereograms was required before the subjects could participate further. The subjects were then shown a card displaying the proper configuration of the fixation and sizecuing dots when properly fused (i.e., $:$ ). A verbal explanation of the correct configuration of the dots accompanied the display card when it was shown to children (i.e., "One in the middle. One 
on the top. One on the bottom. One on one side. One on the other side."). Children were encouraged to accept only the proper dot pattern through use of the following remark: "A boy (girl) told me that he (she) could play this game even if the dots didn't look right. You know what? He (she) got them all wrong!' A photograph was then placed face down in the stereoscope and the subjects were asked if they saw the correct pattern, that is, either $(\because \therefore)$ or $(\cdot)$, depending upon the photograph. If a subject reported that the pattern was not correct, the experimenter adjusted the stereoscope and simultaneously requested the subject to "converge" his/ her eyes until the correct pattern was seen. ${ }^{1}$ When the subject reported the correct pattern, the strobe light was flashed briefly. The subjects were given four practice trials during which exposure duration was progressively decreased to acclimate them to the brief exposures they would experience during testing. During testing, exposure duration was set at 10,4 , and $2 \mathrm{msec}$ for the small, medium, and large stereograms, respectively. These different exposure durations helped compensate for the different spatial frequencies involved, so that stereograms of all three sizes provided about equally effective stimulus contrasts (see Campbell \& Robson, 1968).

Testing began following the practice trials. Each of the 12 photographs was presented 8 times, for a total of 96 trials. The order of presentation was random, with the restriction that each photograph be presented once before any was presented twice, and so forth. Each test trial proceeded in a like manner, with the subject securing proper binocular fusion before the strobe light was illuminated. Five-year-olds were given the option of verbally responding "yes" or "no," or of pointing to replicas of the figure-present and figure-absent displays. All other subjects responded verbally. The subjects were never informed of the accuracy of their judgments.

\section{Instruction}

The subjects were informed that half of the photographs would contain a disk and half would not. They were also told that when one dot appeared on the back of a photograph, the disk, if present, could be any one of the three sizes they had seen previously. When the size-cuing dots were present, they were told that the outer edge of the disk, if present, would touch the four outside dots.

\section{RESULTS}

The data were scored according to the theory of signal detection (Hochhaus, 1972; Swets, Tanner, \& Birdsall, 1961). That is, each response was scored as a hit, a miss, a correct rejection, or a false alarm, and the customary indices of signal detection $\left(d^{\prime}\right)$ and response bias $(\beta)$ were calculated for each subject separately for each of the six experimental conditions: two cue types for each of three disk sizes. ${ }^{2}$

\section{Detectability}

Table 1 shows the mean value and standard deviation of signal detection $\left(d^{\prime}\right)$ for each combination of disk size, cue type, and age group. The larger the value of $d^{\prime}$, the better the performance.

The data summarized in Table 1 were subjected to a $3 \times 3 \times 2$, repeated-measures analysis of variance, with age as a between-subjects effect, and disk size and cue type as within-subjects effects. This analysis yielded four significant effects: a main effect for cue type $[F(1,57)=37.36, p<.01]$; a main effect for disk size $[F(2,114)=5.81, p<.01]$; an age $\times$ cue type interaction $[F(2,57)=5.30, p<.01]$; and a disk size $\times$ cue type interaction $[F(2,114)=13.04, p<.01]$.
Table 1

Values of Signal Detection Bias $\left(d^{\prime}\right)$ as a Function of Age Group, Cue Type, and Disk Size

\begin{tabular}{|c|c|c|c|}
\hline \multirow[b]{2}{*}{ Cue Type } & \multicolumn{3}{|c|}{ Age } \\
\hline & 5 years & 8 years & Adult \\
\hline \multicolumn{4}{|c|}{ Large Disk } \\
\hline \multicolumn{4}{|l|}{ Precued } \\
\hline$M$ & 2.49 & 2.99 & 2.93 \\
\hline$S D$ & 1.13 & .94 & 1.11 \\
\hline \multicolumn{4}{|l|}{ Self-cued } \\
\hline M & 1.67 & 2.40 & 2.51 \\
\hline$S D$ & 1.05 & 1.22 & 1.12 \\
\hline \multicolumn{4}{|c|}{ Medium Disk } \\
\hline \multicolumn{4}{|l|}{ Precued } \\
\hline$M$ & 2.50 & 2.46 & 2.66 \\
\hline$S D$ & .93 & .81 & 1.35 \\
\hline \multicolumn{4}{|l|}{ Self-cued } \\
\hline$M$ & 1.10 & 1.99 & 2.22 \\
\hline$S D$ & .95 & 1.09 & 1.47 \\
\hline \multicolumn{4}{|c|}{ Small Disk } \\
\hline \multicolumn{4}{|l|}{ Precued } \\
\hline$M$ & 2.42 & 2.67 & 2.35 \\
\hline$S D$ & .63 & 1.30 & 1.21 \\
\hline \multicolumn{4}{|l|}{ Self-cued } \\
\hline$M$ & 2.31 & 2.76 & 2.49 \\
\hline$S D$ & 1.10 & 1.10 & 1.25 \\
\hline
\end{tabular}

Note-The larger the value of $d^{\prime}$ the better the performance.

In the main effect of cue type, the disk was generally more detectable on precued $\left(d^{\prime}=2.61\right)$ than on self-cued trials $\left(d^{\prime}=2.16\right)$, as would be expected. In the main effect of disk size, the intermediate size disk was less detectable $\left(d^{\prime}=2.16\right)$ than the other two disks $\left(d^{\prime}=2.50\right.$ for each), but this difference may be trivial because it may only reflect our limited success in equating stimulus contrast for the three disk sizes.

The significant age $\times$ cue type interaction is depicted in Figure 2. The 5-year-olds were more dependent upon the precuing than were their elders. Two additional oneway analyses comparing the three age groups within each of the two cue types yielded a significant effect of age only on self-cued trials $[F(2,57)=3.99, p<.025]$. That is, only on the self-cued trials did the 5-year-olds detect the disks more poorly than did their elders.

The disk size $\times$ cue type interaction is illustrated in Figure 3. Three additional one-way analyses comparing the two cue types for each of the three disk sizes yielded significant effects of cue type for the large disk $[F(1,59)=12.30, p<.01]$ and for the medium sized $\operatorname{disk}[F(1,59)=12.11, p<.01]$, but not for the small disk, whose perimeter lay close to the fixation point. That is, except when the information to be detected was situated close to the point of fixation, precuing proved helpful.

\section{Response Bias}

Table 2 shows the mean value and standard deviation of response bias $(\beta)$ for each combination of disk size, cue type, and age group. The higher the value of $\beta$, the higher the subject's decision criterion; that is, the fewer the false alarms. 


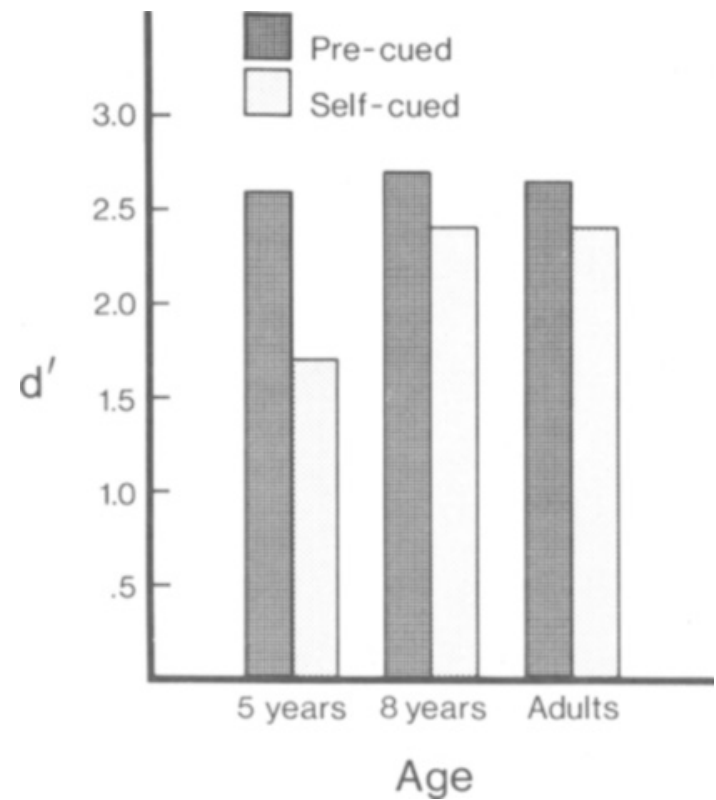

Figure 2. Signal detection $\left(d^{\prime}\right)$ as a function of age group and cue type.

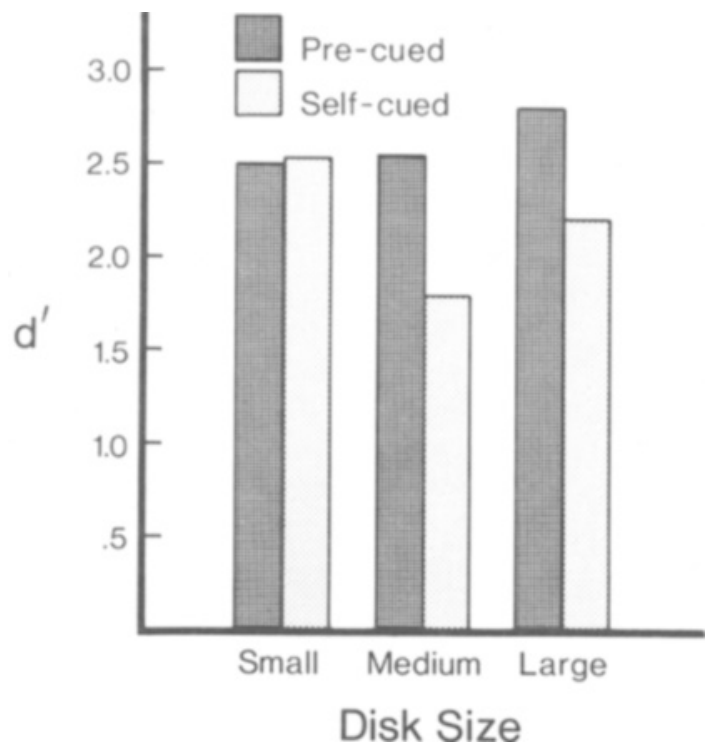

Figure 3. Signal detection $\left(d^{\prime}\right)$ as a function of disk size and cue type. (The small, medium, and large disks subtended visual angles of $3^{\circ}, 5^{\circ}$, and $8^{\circ}$, respectively.)

The data summarized in Table 2 were subjected to a three-way analysis of variance, which yielded three significant effects: a main effect for age $[F(2,57)=9.19$, $p<.01]$, an age $\times$ disk size interaction $[F(4,114)=$ $6.63, p<.01]$, and a disk size $\times$ cue type interaction $[F(2,114)=6.28, p<.01]$.

In the main effect of age, the 5-year-olds' average decision criterion $(\beta=3.04)$ was more stringent than the adults' $(\beta=1.67)$, with the 8-year-olds' average ( $\beta=2.62)$ falling in between. However, this main effect is sharply qualified by the age $\times$ disk size interaction, illustrated in Figure 4, which shows that the 8-year-olds' choice of $\beta$ varied markedly from one disk size to another. Additional one-way analyses of variance comparing the three disk sizes within each age group yielded a significant effect of disk size only for the 8-year-olds $[F(2,114)=6.28, p<.01]$. As illustrated in Figure 4, the 8-year-olds' decision criterion was exceptionally high for the intermediate size disk; otherwise, the data are consistent with the main effect noted above.

The disk size $\times$ cue type interaction is illustrated in Figure 5. Three additional one-way analyses of variance comparing the two cue types for each disk size yielded a significant difference only for the large disk $[F(1,59)=$ $6.56, p<.025]$. That is, only when the information to be detected was far from the fixation point did the subjects adopt a more stringent decision criterion on self-cued trials $(\beta=2.71)$ than on precued trials $(\beta=1.84)$.

\section{DISCUSSION}

The subjects in the present experiment were required to do two things at once on each trial: (1) binocularly fuse a pair of dichoptic fixation points, and (2) detect, during a tachistoscopic exposure, the presence or absence of a stereoscopic disk whose perimeter lay $1.5^{\circ}, 2.5^{\circ}$, or $4^{\circ}$ from the fused fixation point. The task was designed to

Table 2

Values of Response Bias $(\beta)$ as a Function of Age Group, Cue Type, and Disk Size

\begin{tabular}{cccc}
\hline & \multicolumn{3}{c}{ Age } \\
\cline { 2 - 4 } Cue Type & 5 years & 8 years & Adult \\
\hline \multicolumn{3}{c}{ Large Disk } \\
Precued & \multicolumn{3}{c}{} \\
$M$ & 2.33 & 2.05 & 1.15 \\
$S D$ & 1.80 & 1.68 & 1.08 \\
Self-cued & & & \\
$M$ & 3.56 & 2.48 & 2.10 \\
$S D$ & 2.12 & 1.90 & 1.76 \\
& Medium Disk & & \\
Precued & & & \\
$M$ & 2.80 & 3.65 & 1.11 \\
$S D$ & 1.98 & 1.75 & .81 \\
Self-cued & & & \\
$M$ & 2.74 & 3.56 & 2.11 \\
$S D$ & 1.97 & 1.91 & 1.67 \\
& Small Disk & & \\
Precued & & & 1.83 \\
$M$ & 3.81 & 2.09 & 1.42 \\
$S D$ & 1.97 & 1.65 & 1.70 \\
Self-cued & & 1.92 & 1.21 \\
$M$ & 3.04 & 1.43 & \\
$S D$ & 2.05 & & \\
\hline
\end{tabular}

Note-The larger the value of $\beta$ the more conservative the decision criterion (e.g., fewer false alarms). 


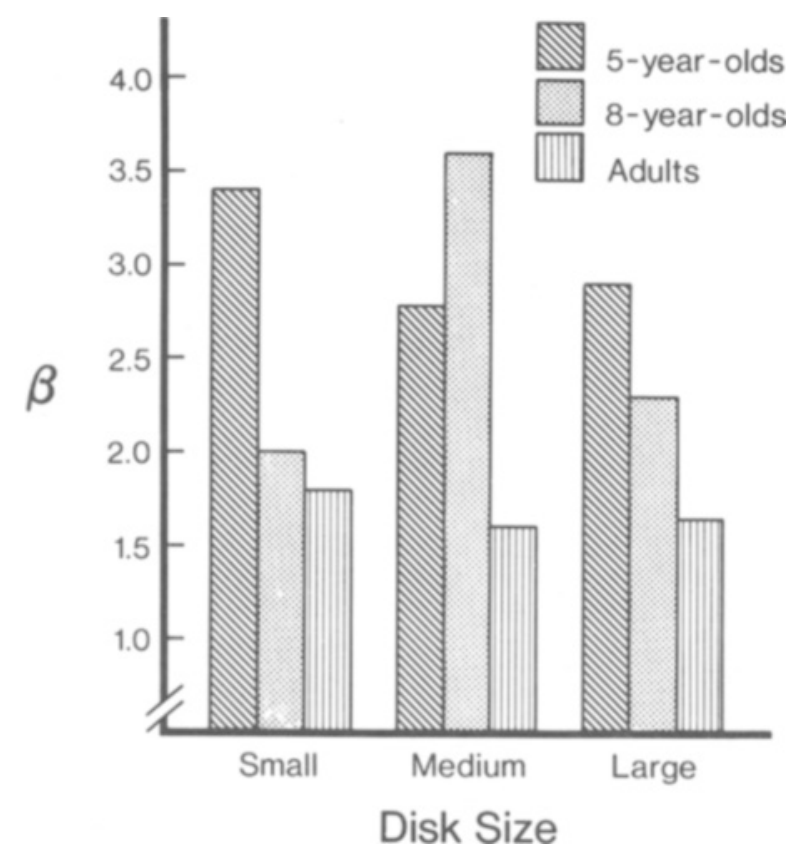

Figure 4. Response bias $(\beta)$ as a function of age group and disk size. (The small, medium, and large disks subtended visual angles of $3^{\circ}, 5^{\circ}$, and $8^{\circ}$, respectively.)

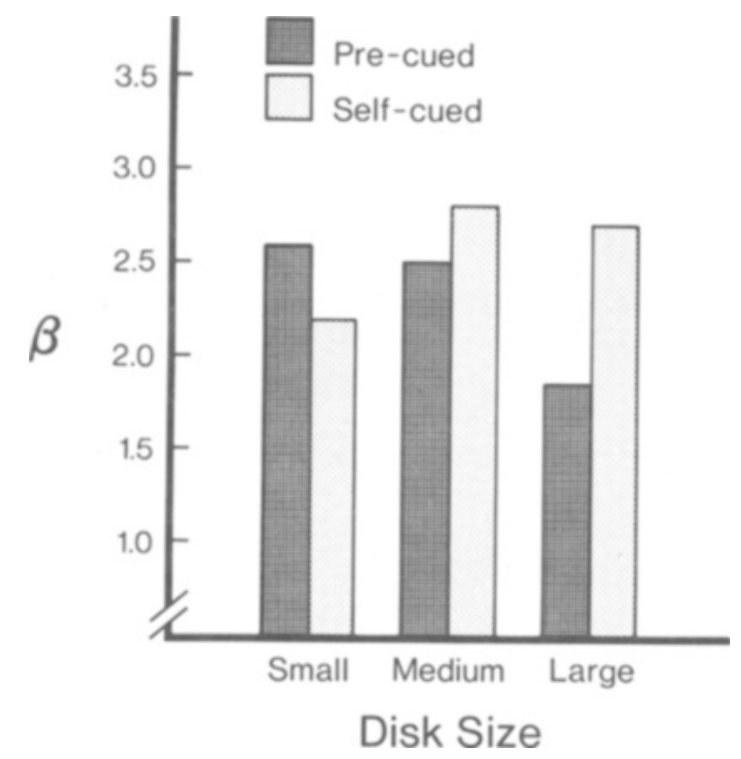

Figure 5. Response bias $(\beta)$ as a function of disk size and cue type. (The small, medium, and large disks subtended visual angles of $3^{\circ}$, $5^{\circ}$, and $8^{\circ}$, respectively.)

favor subjects according to their ability to allocate their visual attention selectively; that is, according to their ability to look in one direction while gazing in another.

In order to obtain a quantitative index of signal detection $\left(d^{\prime}\right)$ independent of the subject's response bias $(\beta)$, each subject's raw data was scored according to the theory of signal detection (Swets, 1973). Although our interest in $\beta$ was incidental to our interest in $d^{\prime}$, we will discuss both sets of data in some detail, beginning with the latter.

\section{Signal Detection}

The major result of the present study was the finding that precuing subjects explicitly as to the location of the disk's perimeter improved signal detection more for the 5-year-olds than for their elders. When subjects were forewarned of the location of the impending disk's perimeter (precued trials), neither the 8-year-olds nor the adults performed reliably better than the 5-year-olds. However, when no such attentional aids were provided (self-cued trials) the 5-year-olds performed more poorly than both the 8-year-olds and the adults, who performed alike. We found, therefore, that 5-year-olds are capable of allocating their visual attention selectively, just as are adults, even though they do not deploy attention as quickly or as effectively as their elders when the focus of attention is theirs to choose.

This finding is consistent with the results of several other studies that have found no developmental differences in the ability to allocate attention selectively during tachistoscopic exposure (Cohen \& Haith, 1977; Lane \& Pearson, 1983; Miller, 1971, 1973; Stewart-Lester \& Lefton, 1981). However, only in the present study was the direction of the subject's gaze precisely controlled, so the similarity of results in these experiments may be more apparent than real. Some contrary evidence comes from studies that required subjects to recognize letters of the alphabet, a skill known to vary with age (Fisher \& Lefton, 1976; Taylor, 1982). Since differential reading skill may account for the observed developmental effects, it does not appear warranted to attribute them to visual attention per se. That is, to date there appears to be no good experimental evidence that the rudimentary capacity for selective visual aftention develops beyond the age of 5 years.

What does appear to develop beyond the age of 5 years is the skillful utilization of this visual ability (e.g., speed of deployment). For instance, in the present experiment the perimeter of the random-dot array provided a very salient, although indirect, cue as to the approximate location of the perimeter of the corresponding disk whose presence or absence was to be detected. The 8-year-olds and adults appear to have used this intrinsic cue to aim their attention toward the disk's perimeter as soon as the outline of the random-dot array became visible. The 5-year-olds, on the other hand, do not appear to have shifted their attention from the fused fixation point during self-cued trials and, therefore, were ill prepared to detect any disk except the small one, which they detected about as well as did their elders.

This interpretation is consistent with the significant disk size $\times$ cue type interaction, a finding which also suggests that the perimeter of the small disk could be effectively detected without the subjects' shifting their attention from the fixation dot. The smallest of the three disks was also presented for a longer period of time during each trial, but this cannot reasonably account for the 5-year-olds' 
success in detecting the smallest disk in the absence of attentional precuing. The temporal resolution of photopic vision (i.e., its integration time) is at least $20 \mathrm{msec}$, well above all three of the stimulus durations employed $(2,4$, and $10 \mathrm{msec}$ ). Furthermore, visual attention is generally presumed to scan the visual remanence of a tachistoscopic stimulus, and this remanent icon outlasts the integration interval by approximately $250 \mathrm{msec}$ (see Haber \& Hershenson, 1973, p. 168). In the present experiment, the salience of the remanent icons were equated across the three stimulus sizes, at least in some approximation, by offsetting the stereograms' different spatial frequencies with different tachistoscopic durations.

Both the 8-year-olds and the adults appear to have had enough time during the iconic interval to shift their attention to the perimeter of even the largest of the three disks. This is not surprising given the speed at which visual attention is said to shift. For example, Tsal (1983) has estimated that it takes approximately $8 \mathrm{msec}$ per degree of visual angle, which means that a shift of $4^{\circ}$, the radius of our largest disk, would have required only $32 \mathrm{msec}$. Although the precision of Tsal's estimate is in dispute (Eriksen \& Murphy, 1987), there appears to be no doubt that visual attention may be shifted within the time span of visual remanence. Therefore, what is particularly interesting about our results is that 5-year-olds, who showed that they were capable of deploying their visual attention effectively when attentional precuing was provided, did not appear to do so when cues were not explicit or provided in advance. Apparently, when the focus of attention is theirs to choose, 5-year-olds require more time than their elders to shift their visual attention, either because the shift itself occurs more slowly or because the shift has a longer latency. This latency may reflect the 5-yearolds' limited skill in selecting informative regions of the icon at which to direct their attention when no explicit cues are provided.

The present results, specifically the significant effect of age on the self-cued trials, appear similar to those reported by Holmes, Cohen, Haith, and Morrison (1977). They found that adults were more capable than children (5- and 8-year-olds) in identifying a geometric form imaged tachistoscopically upon the periphery of the retina a fixed distance from the fovea in one of eight unpredictable directions. Furthermore, the performance means of the 8-year-olds in their study were intermediate to those of the other two groups at each of the retinal eccentricities tested: $1^{\circ}, 2^{\circ}, 4^{\circ}$, and $6^{\circ}$. Moreover, at $2^{\circ}$ and $4^{\circ}$, the two target distances that fell within the range used in the present study, the 8-year-olds' means were closer to the adults' than to the 5-year-olds', as was the case in the present experiment.

Sheingold (1973) has also reported a similar difference between 5-year-olds and their elders in a partial report task. In that study, the subject was to identify the one form, from among eight geometric forms circularly arrayed about a central fixation point, that was indicated by a single, simultaneous, intrinsic pointer (Sheingold, 1973, Figure 3, Condition S). Sheingold found that 5-year-olds did more poorly than 8-year-olds, who did as well as older subjects. Of course, the partial-report paradigm confounds visual attention and visual memory, among other things, and the similarity in findings may, therefore, be more apparent than real.

\section{Response Bias}

The decision criterion $(\beta)$ of the ideal observer depends upon (1) the a priori probability of signal occurrence and (2) the value and cost of correct and incorrect judgments, respectively. In the present experiment, wherein the a priori probability of signal occurrence was objectively .5 and the value and cost of correct and incorrect judgments were objectively equal (and opposite), the ideal observer's decision criterion would have been $\beta=1$. The adults' average was closer to this ideal, but the average value of $\beta$ utilized by each age group, adults included, was substantially greater than $\beta=1$. That is, each age group adopted a stringent decision rule which minimized false alarms at the expense of hits. Either the subjects underestimated the objective probability of signal occurrence or they were subjectively more concerned with minimizing errors of commission (false alarm) than they were of errors of omission (miss).

\section{REFERENCES}

CAmpbell, F. W., \& Robson, J. B. (1968). Application of Fourier analysis to the visibility of gratings. Journal of Physiology, 197, 551-566.

CoHEN, K. M., \& HaITH, M. M. (1977). Peripheral vision: The effects of developmental, perceptual, and cognitive factors. Journal of Experimental Child Psychology, 24, 373-394.

EruKsen, C. W., \& MurPhy, T. D. (1987). Movement of attentional focus across the visual field: A critical look at the evidence. Perception \& Psychophysics, 42, 299-305.

Fisher, D. F., \& Lefton, L. A. (1976). Peripheral information extraction: A developmental examination of reading processes. Journal of Experimental Child Psychology, 21, 77-93.

Haber, R. N., Hershenson, M. (1973). The psychology of visual perception. New York: Holt, Rinehart \& Winston.

HELMHOLTZ, H. VON (1962). Helmholtz's treatise on physiological optics (J. P. C. Southall, Ed. and Trans.) (Vol. 3). New York: Dover Publications. (Original work published 1925)

Hochraus, L. (1972). A table for the calculation of $d^{\prime}$ and $\beta$. Psychological Bulletin, 77, 357-376.

Holmes, D. L., Cohen, K. M., Haith, M. M., \& Morrison, F. J. (1977). Peripheral visual processing. Perception \& Psychophysics, 22, 571-577.

Julesz, B. (1971). Foundations of cyclopean perception. Chicago: University of Chicago Press.

Lane, D. M., \& Pearson, D. A. (1983). Attending to spatial locations: A developmental study. Child Development, 54, 98-104.

MiLLER, L. K. (1971). Developmental differences in the field of view during tachistoscopic presentation. Child Development, 42, 1543-1551.

Miller, L. K. (1973). Developmental differences in the field of view during covert and overt search. Child Development, 44, 247-252.

MüLleR, H. J., \& FindLAY, J. M. (1987). Sensitivity and criterion effects in the spatial cuing of visual attention. Perception \& Psychophysics, 42, 383-399. 
SHEINGOLD, K. (1973). Developmental differences in intake and storage of visual information. Journal of Experimental Child Psychology, 16, $1-11$.

STEWART-Lester, K. J., \& LefTon, L. A. (1981). Information extraction from the parafovea: A developmental study. Journal of Experimental Psychology: Human Perception \& Performance, 7, 624633.

SwETs, J. A. (1973). The relative operating characteristic in psychology. Science, $182,990-1000$.

Swets, J. A., Tanner, W. P., Birdsall, T. B. (1961). Decision processes in perception. Psychological Review, 68, 301-340.

TAYLOR, H. G. (1982). Age differences in peripheral letter perception. Joumal of Experimental Psychology: Human Perception \& Performance, 8, 106-112.

TSAL, Y. (1983). Movements of attention across the visual field. Journal of Experimental Psychology: Human Perception \& Performance, 9, 523-530.

\section{NOTES}

1. The subjects had more difficulty fusing the two fixation dots when they were accompanied by their respective attention-delimiting dots. Subjects would sometimes fuse the left fixation dot with one of the attention-delimiting dots that flanked the right fixation dot. By monitoring binocular fusion on each trial, we were able to correct such errors before presenting the stimulus.

2. Small-sample estimates of parameters $d^{\prime}$ and $\beta$ are particularly variable when hit rates approach 1 or false alarm rates approach 0 , as was typically the case in the present experiment. To lessen this variability, a frequency of 1 was added to the tally of each subject's judgments within each of the six experimental conditions, with $1 / 4$ going into each cell of the matrix: hit, miss, correct rejection, and false alarm.

(Manuscript received April 11, 1988; revision accepted for publication October $21,1988$. ) 